\title{
Accelerating Nearest Neighbor Search on Manycore Systems
}

\author{
Lawrence Cayton \\ Max Planck Institute \\ Tübingen, Germany \\ Icayton@tuebingen.mpg.de
}

\begin{abstract}
We develop methods for accelerating metric similarity search that are effective on modern hardware. Our algorithms factor into easily parallelizable components, making them simple to deploy and efficient on multicore CPUs and GPUs. Despite the simple structure of our algorithms, their search performance is provably sublinear in the size of the database, with a factor dependent only on its intrinsic dimensionality. We demonstrate that our methods provide substantial speedups on a range of datasets and hardware platforms. In particular, we present results on a 48-core server machine, on graphics hardware, and on a multicore desktop.
\end{abstract}

\section{INTRODUCTION}

We study methods to accelerate nearest neighbor search on modern parallel systems. We work in the standard metric nearest neighbor (NN) setting: given a database $X$, return the closest point to any query $q$, where closeness is measured with some fixed metric. Though the problem setting is by now well-studied, there are two recent developments that provide a different focus for this work. The first development comes from studies in data analysis and advances in theoretical computer science, where the notion of intrinsic dimensionality has been refined and profitably exploited to manage high-dimensional data. The second development comes from the computer hardware industry: CPUs are virtually all multicore now and GPUs have rapidly evolved into powerful secondary devices for numerical computation, forcing a renewed interest in parallelism.

High-dimensional data challenges nearly all methods for accelerating NN search. Unfortunately, complicated, highdimensional data is the norm in many domains that rely on NN search, such as computer vision 28], bioinformatics 4], and data analysis in general 12 . Because such data must be dealt with, researchers have explored data properties that might be exploited to accelerate search. A compelling property is the intrinsic dimensionality of a data set; the idea is that often data only appears high-dimensional (i.e., each el-

Permission to make digital or hard copies of all or part of this work for personal or classroom use is granted without fee provided that copies are not made or distributed for profit or commercial advantage and that copies bear this notice and the full citation on the first page. To copy otherwise, to republish, to post on servers or to redistribute to lists, requires prior specific permission and/or a fee.

Copyright 20XX ACM X-XXXXX-XX-X/XX/XX ...\$10.00. ement has many features), but is actually governed by only a few parameters. In recent years, this type of data has been studied extensively and is now believed to be widespread; in machine learning, for example, several methods have been developed to reveal the intrinsic structure of data 26, 27. In research on accelerated NN retrieval, a renewed focus on intrinsic dimensionality in recent years has yielded methods with strong theoretical guarantees 18,20 and state of the art empirical performance 2 .

The end goal of all of this work on $\mathrm{NN}$ is, of course, to make NN search run fast. While properties of data are important for computational efficiency, equally important is the machine hardware on which search actually runs. Hardware properties are especially important now, as the machines in use for everyday data analysis and database operations are fundamentally different than they were even a decade ago. In particular, standard computer chips are multicore with vector (SIMD) units, and GPUs have become popular as secondary devices for data-intensive processing. These modern processors have very impressive computational capabilities, but fully exploiting it requires considerable parallelism.

The shift towards parallelism in hardware necessitates a refocusing in algorithm design and software engineering methods 24. At the level of algorithm design, advances in algorithms and data structures may not be useful unless they can be effectively parallelized. At the level of software development, developing efficient implementations on parallel systems is challenging, so parallel primitives and design patterns are required to ease the burden on programmers. For the case of NN search, the modern search algorithms discussed were developed for sequential systems, and seem quite difficult to deploy on modern hardware. This is a major practical limitation.

In this work, we develop an new approach to NN search that is both provably sensitive to intrinsic structure and that is effective on modern CPUs and GPUs. Our algorithms are based on fundamental ideas from metric similarity search, and are designed carefully for two important goals. First, our design choices allow us to rigorously bound the search complexity of our methods. Equally important, these choices allow us to factorize our algorithms into a basic primitive that is simple to parallelize, making them effective and relatively easy to implement on different systems.

Our methods are of immediate practical use. We demonstrate their performance benefits on a range of modern platforms: a 48-core server machine, a GPU, and a multicore desktop. 


\section{BACKGROUND AND RELATED WORK}

The focus of the present work is unique; as far as we know, it is the only work to simultaneously make use of modern algorithmic developments and modern hardware. Still, the ideas behind our methods are based on a substantial amount of previous work: the algorithmic ideas are based on techniques from metric similarity search; the results on intrinsic dimensionality are related to ideas developed mostly in the theory and data analysis communities; and the motivation behind the project comes from recent trends in computer hardware, especially as related to databases and scientific computation.

The data structure and algorithm in this work are based on two fundamentals of algorithms for metric data: space decomposition and the triangle inequality. These pillars are used in virtually all work on metric NN search; see the surveys of Chávez et al. and Clarkson for detailed overviews 8, 10. Two of the most empirically effective structures are AESA 29] and metric ball trees 23, 31], both of which have spawned many relatives.

A long-standing problem in similarity search is the difficulty of dealing with high-dimensional data; see [1, 3, 30. and the above surveys. The basic challenge is that spacedecomposition structures that reduce the work for NN retrieval seem to have performance that scales exponentially with the dimensionality of the data, rendering them useless to all but the smallest problems. Within the last two decades there have been two very promising directions of work that attempt to deal with the problem of high-dimensional data.

The first is called Locality Sensitive Hashing (LSH) 16]. LSH has retrieval performance that is provably sublinear, independent of the underlying dimensionality. This was a major theoretical breakthrough and the data structure has been successfully deployed on some tasks (e.g. 4]). However, LSH has some limitations: it can only provide approximate answers, it is defined only for particular distance measures (not at the generality of metrics), and setting the parameters correctly can be complex 11 .

The second line of work, upon which we build, is based on the notion of intrinsic dimensionality. The basic idea here is that many data sets only appear high-dimensional, but are actually governed by a small number of parameters. Within data analysis and machine learning, the idea of lowdimensional intrinsic structure has become extremely popular and such structure is believed to be common in many data sets of interests 26, 27.

This idea has also been explored in the context of NN search. A variety of slightly different notions of metric space dimensionality capture this intuition. One which has recently resulted in strong theoretical and empirical results is the growth dimension or expansion rate 9, 18, which we define formally later. This notion of dimension lead to the development of the Cover Tree [2, 20, which we return to momentarily. Though the notion has some idiosyncrasies [20], the impressive empirical performance of the Cover Tree suggests that it is a useful notion.

Perhaps the two most relevant methods for NN search are the Cover Tree and the GNAT of Brin [3]; let us distinguish this research from the present work. The GNAT uses a simple space decomposition based on representatives from the database, much as we do, and also discusses the idea of intrinsic dimensionality. However, the relationship of the GNAT's search performance and the intrinsic dimensionality is only discussed in an informal, heuristic way, whereas we give rigorous runtime guarantees. These rigorous bounds require a search algorithm that is different than that of the GNAT. Additionally, parallelization is not discussed in 3].

The Cover Tree has rigorous guarantees on the query time dependent on the expansion rate and has empirical performance that is state of the art. Even so, our algorithms, data structure, and theory are substantially different; in particular, the Cover Tree is a deep tree and is explored in a conditional way that seems quite difficult to parallelize. We compare our method with the Cover Tree in the experiments.

Lastly, we touch on the major inspiration for this paper: the use of hardware to accelerate data-intensive processes. Impelled by the sudden ubiquity of multicore CPUs and the development of GPUs for general-purpose computation, this area of research has exploded in the last decade; let us provide a couple of inspiring examples. A relatively early work develops methods to off-load expensive database operations onto the GPU 15. A very recent piece of work tunes basic tree search algorithms (such as for index lookup) to be effective on modern multicore CPUs and GPUs [19]. Finally, another paper suggests simply running brute force search on a GPU to accelerate NN search [5] ; this simple approach provides a surprising amount of acceleration over computation on sequential CPUs 14 .

\section{THE BRUTE FORCE PRIMITIVE}

Brute force search requires a high amount of work, but parallelizes effectively. In contrast, most accelerated approaches to NN search reduce the work, but seem difficult to parallelize. Our approach to NN search takes the advantages of both: it reduces the work and parallelizes effectively. We achieve this combination by structuring our algorithms as multiple (actually two) brute force searches, each of which considers only a small portion of the database. In this section, we formalize the brute force primitive and discuss the parallelization of it.

Given a set of queries $Q$ and a database $X$ with $n$ elements, finding the NNs for all $q \in Q$ can be achieved by a series of linear scans. For each query $q$, the distance between $q$ and each $x \in X$ is computed, the distances are compared, and the database point that is closest is returned. We denote this subroutine as $\mathbf{B F}(Q, X)$. If $L$ is some sets of IDs (i.e. $L \subset\{1, \ldots, n\}$ ), then brute force search between $Q$ and this subset of the database is denoted $\mathbf{B F}(Q, X[L])$.

The work required for $\mathbf{B F}(Q, X)$ is $O(n)$; we later prove that our search algorithms have work only roughly $O(\sqrt{n})$, which is performed in two brute force calls $\mathbf{B F}\left(Q, X\left[L_{1}\right]\right)$ and $\mathbf{B F}\left(Q, X\left[L_{2}\right]\right)$, where lists $L_{1}$ and $L_{2}$ are determined by our algorithm.

Parallelizing $\mathbf{B F}(Q, X)$ is straightforward. We break the procedure down into two steps: a distance computation step, and a comparison step. In the distance computation step, all pairs of distances are computed. This has virtually the same structure as matrix-matrix multiply, and hence block decomposition approaches are effective. In the case where there is only a single query presented at a time (e.g. a stream of queries), the distance computation step of $\mathbf{B F}(q, X)$ has the structure of a matrix-vector multiplication. In both cases, the parallelization is extremely well-studied.

The second step is the comparison: for each query, the distances must be compared, and the nearest database element returned. This is simple to do in parallel systems 
as well; the problem can simply be plugged into the standard parallel-reduce paradigm where comparisons are made according to an inverted binary tree.

The computational structure of accelerated NN data structures is quite different. For simplicity, let us take metric trees as an example 23. Querying this data structure requires a depth-first search of a deep tree. This process involves an interleaved series of distance computations, bound computations, and distance comparisons. Moreover, the computation is conditional: the specific calculations made at one step depend on the comparisons from the previous step. Distributing the work for this process across processors is a significant challenge. Additionally, because of the interleaved nature of the computation, attaining near fullutilization of the hardware is difficult; in contrast, matrixmatrix multiply is commonly used to demonstrate the capability of processors. Finally, the conditional nature of the computation makes execution on vector hardware with limited branching abilities - namely, GPUs - inefficient.

Efficient NN routines seem to depend on a complex computational structure; a major contribution of the present work is in demonstrating that a much simpler structure can be substituted without significant loss.

With the brute force primitive in place, we proceed to discuss our data structure and algorithms, all of which will be built from this primitive.

\section{DATA STRUCTURE}

We discuss the data structure underlying our methods in this section, which we call the Random Ball Cover (RBC). This is a very simple, single-level cover of the underlying metric space. The basic idea is to use a random subset of the database as representatives for the rest of the DB. The details of this structure differ slightly for our two different search algorithms, which will be introduced in the next section.

The database is denoted $X=\left\{x_{1}, \ldots, x_{n}\right\}$ and the metric in use is denoted $\rho(\cdot, \cdot)$. The data structure consists of a random subset of the database, which will generally be of size about $O(\sqrt{n})$; we make this precise later. This set of random representatives will be denoted $R$. It is built by choosing each element of the database independently at random with probability $n_{r} / n$, where the exact value of $n_{r}$ is discussed in the theory section. In expectation, there will be $n_{r}$ representatives chosen - one can think of the symbol $n_{r}$ as shorthand for number of representatives.

Each representative owns some subset of the database. The list of points that a representative $r$ owns is denoted $L_{r}$. In the exact search algorithm, $L_{r}$ contains all $x \in X$ for which $r$ is the nearest neighbor among $R$. In the one-shot algorithm, $L_{r}$ contains a suitably sized set of $r$ 's NNs among $X$. We will at times refer to $L_{r}$ as an ownership list.

Along with each representative, a radius is also stored. This radius is defined as the distance from $r$ to the furthest point that it owns:

$$
\psi_{r}=\max _{x \in L_{r}} \rho(x, r)
$$

The focus of this paper is on algorithms that are simple to parallelize; this is achieved by folding the computational work into brute force calls. The building routines demonstrate this principle concisely. The building routine for the exact search algorithm must find the NN for each $x \in X$ among the representatives $R$. Thus this routine is simply a call to $\mathbf{B F}(X, R)$. Similarly, the building routine for the one-shot algorithm must find the $\mathrm{NN}(\mathrm{s})$ for each representative $R$ among the database elements $X$; thus this procedure is simply a call to $\mathbf{B F}(R, X)$. Both parallelize easily.

With the data structure and notation in place, we proceed to describe the search algorithms.

\section{SEARCH ALGORITHMS}

In this section, we describe two search algorithms which use the RBC data structure. The theoretical analysis of these algorithms appears in the next section. Both of these algorithms build up from the brute force search primitive.

We first describe the one-shot algorithm, then the exact search algorithm. The one-shot algorithm is extraordinarily simple, the exact algorithm only slightly less so. Both algorithms rely on a randomized data structure, and so are probabilistic. In the one-shot algorithm, the solution itself is randomized: the data structure returns a correct result with high probability. In the exact algorithm, the solution is guaranteed to be correct; only the running time is probabilistic ${ }^{1}$ Hence when an exact answer is required, the exact algorithm is appropriate; if a small amount of error can be tolerated, the one-shot algorithm is simpler and often faster, as we show in the experiments.

We focus on the problem of 1-NN search throughout; the extensions to $k$-NN and $\epsilon$-range search are straightforward.

\subsection{One-shot search}

First, recall the data structure built for the one-shot search algorithm. The representatives are chosen at random, and each list $L_{r}$ contains the $s$ closest database elements to $r$. Depending on the setting of $s$, points will typically belong to more than one representative. We discuss these parameters further in the theory section.

On a query $q$, the algorithm proceeds as follows. It first computes the NN to $q$ among the representatives using a simple linear scan (brute force search), call it $r$. It then scans the ownership list $L_{r}$, computing the distance from $q$ to each listed database point. The nearest one is returned as the nearest neighbor.

We restate the algorithm in terms of the brute force primitive. The algorithm first calls $\mathbf{B F}(q, R)$, which returns a representative $r$. It then calls $\mathbf{B F}\left(q, X\left[L_{r}\right]\right)$ and returns the answer.

The one-shot algorithm is almost absurdly simple. Yet, as we show rigorously in the theory section, it provides a massive speedup; in particular, it reduces the work from $O(n)$ (required for a full brute force search) to roughly $O(\sqrt{n})$. Moreover, it is very fast empirically, as we show in the experiments section.

\subsection{Exact search}

Whereas the one-shot algorithm does not use the triangle inequality (though the analysis requires it), the exact search algorithm explicitly prunes portions of the space using it; in this sense, it is reminiscent of classic branch-and-bound techniques.

Recall that the data structure built for the exact search algorithm is slightly different from the one for the one search

\footnotetext{
${ }^{1}$ This algorithm can be easily modified so that it only guarantees an approximate nearest neighbor, which reduces search time.
} 
algorithm (the reason for the difference will become clear in theory section). The build algorithm calls $\mathbf{B F}(X, R)$, then adds each $x \in X$ to the ownership list of its returned NN in $R$. The radii $\psi_{r}$ are set to $\max _{x \in L_{r}} \rho(x, r)$ as before.

We now detail the search algorithm. First, the closest point to $q$ among all $r \in R$ is computed; call it $r_{q}$, and let $\gamma=\rho\left(q, r_{q}\right)$. This distance is an upper bound on the distance to $q$ 's NN (since $r_{q} \in X$ ), and so the algorithm can use it to discard some of the database from consideration. Recall that the radius of each representative $r$ is stored as $\psi_{r}-i$.e. each $x \in L_{r}$ satisfies $\rho(x, r) \leq \psi_{r}$. Because $\gamma$ is an upper bound on the distance to the NN, any point belonging to an $r$ satisfying

$$
\rho(q, r) \geq \gamma+\psi_{r}
$$

can be discarded. The following sketch illustrates (1); since there is a point within distance $\gamma$ of $q$, no points within distance $\psi_{r}$ of $r$ can be $q$ 's NN.

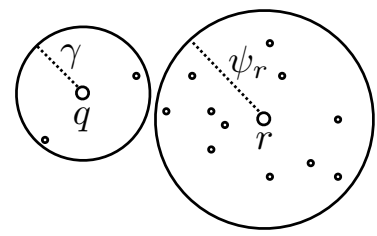

Hence the only points that need to be considered belong to a list $L_{r}$, where $r$ violates (1).

The algorithm simultaneously checks a second bound in hopes of pruning out more of the representatives. As Lemma 1 (see the next section) shows, if $r_{q}$ is $q$ 's NN among the representatives, any representative that owns $q$ 's NN must satisfy

$$
\rho(q, r) \leq 3 \cdot \rho\left(q, r_{q}\right) .
$$

Hence any representative violating this inequality is pruned out by the search algorithm.

Once the pruning stage is complete, the search algorithm computes the distance to all points belonging to one of the remaining representatives, and returns the nearest.

We restate the algorithm in terms of our primitive. It first computes $\mathbf{B F}(q, R)$, much like the one-shot algorithm. In this case, however, the distances must be retained so that inequalities (1) and 22 can be checked. Once the inequalities are checked, some representatives will still remain, with lists $L_{1}, \ldots, L_{t}$. Next the search algorithm performs another brute force search, namely $\mathbf{B F}\left(q, X\left[L_{1} \cup L_{2} \cup \ldots \cup L_{t}\right]\right)$, and returns the answer.

We will see that the size of each of the brute force calls is about $O(\sqrt{n})$, providing major time savings over a full brute force search.

We emphasize that the computation structure of both search algorithms is quite different from tree-based search, in which bounds are incrementally refined, and distance computations are interleaved with bound evaluations. In both cases, this structure makes the algorithm extremely simple to implement and effective to parallelize. It is rather surprising that such simple algorithms can effectively reduce the work required for NN search, but that is exactly what we show both theoretically and empirically in the following sections.

\section{THEORY}

As we described in the background section, all methods that reduce the work for NN search have some dependence on the dimensionality of the database. Much of the success of metric indexing methods is commonly ascribed to their dependence only on the intrinsic dimensionality of data. In this section, we prove that the RBC search algorithms scale with the expansion rate, which is a useful notion of intrinsic dimensionality.

Definition 1. Let $B(x, r)$ denote the closed ball of radius $r$ around $x$-i.e. the set $\{y: \rho(x, y) \leq r\}$. A finite metric space $M$ has expansion rate $c$ if for all $r>0$ and $x \in M$

$$
|B(x, 2 r)| \leq c \cdot|B(x, r)| .
$$

To gain some intuition for this measure, consider a grid of points in $\mathbb{R}^{d}$ under the $\ell_{1}$ metric

$$
\rho(x, y)=\sum_{i=1}^{d}\left|x_{i}-y_{i}\right| .
$$

The expansion rate in this case is $2^{d}$, hence $\log c$ corresponds to the dimensionality of the data 18. Notice that the expansion rate is defined only in terms of the metric, not in terms of the representation of data; in this sense, the rate captures the intrinsic structure of the metric space. Notice also that the expansion rate is defined for arbitrary metric spaces, so makes sense for the edit distance on strings and the shortest path distance on the nodes of a graph, for example.

Throughout we assume that $X \cup Q$ has expansion rate $c$, and we prove bounds dependent on this expansion rate and $n$.

The exact search algorithm and analysis rely on the following lemma, which is known [10. See Appendix A for the proof.

Lemma 1. Let $R \subset X$ and assign each $x \in X$ to its nearest $r \in R$. Let $\gamma=\min _{r \in R} \rho(q, r)$ (i.e., $\gamma$ is the distance to $q$ 's $N N$ in $R$ ). Then, if some $r^{*} \in R$ owns the nearest neighbor to $q$ in $X$, it must satisfy

$$
\rho\left(q, r^{*}\right) \leq 3 \gamma
$$

We now analyze the search algorithms. Throughout, we assume $R$ is a random subset of $X$, built by picking each element of $X$ independently at random with probability $n_{r} / n$. Sometimes we will denote this probability as $p$. Recall that the ownership list of $r \in R$ is denoted $L_{r}$ and the radius of this list (i.e. $\max _{x \in L_{r}} \rho(x, r)$ ) is denoted $\psi_{r}$. Finally, $n_{r}$ is the expected number of representatives and $n$ is the cardinality of the database.

\subsection{Exact Search}

First, let us consider the exact search algorithm. The search algorithm performs two steps: in the first step, the algorithm performs brute force search from the queries to $R$; and in the second step, it performs a brute force search from the queries to the database elements belonging to ownership lists of un-pruned representatives. The first step clearly has work complexity $O\left(n_{r}\right)$ per query, where $n_{r}$ is the expected number of representatives; the following analysis bounds the complexity of the second step. In particular, we show 
that the expected number of distance evaluations is $c^{3} n / n_{r}$. Hence if $n_{r} \approx c^{3 / 2} \sqrt{n}$, the expected number of distance evaluations in the second step is $O\left(c^{3 / 2} \sqrt{n}\right)$, the same as the first step. We call $n_{r}=O\left(c^{3 / 2} \sqrt{n}\right)$ the standard parameter setting.

In the first step of the algorithm, the nearest point to $q$ in $R$ is found; call this point $r_{q}$. How many database points are likely to be closer to $q$ than $r_{q}$ ?

Claim 1. Let $\gamma$ be the distance from $q$ to its nearest neighbor in $R, r_{q}$. The expected number of points in $B(q, \gamma)$ is $n / n_{r}$, which is $O\left(\sqrt{n} / c^{3 / 2}\right)$ for the standard parameter setting.

Proof. Form a list $L=\left[x_{1}, x_{2}, \ldots, x_{n}\right]$ by ordering the database points $x \in X$ by their distance to $q$. Some subset of $X$ also belongs to $R$; let $x_{t}$ be the first representative appearing in $L$ (i.e. the closest representative to $q$ ). Then the expected number of points in $B(q, \gamma)$ is equal to $t-1$.

A slightly different way to view the process is that the $L$ is fixed, then $x_{1}$ is chosen as a representative with probability $n_{r} / n$, then $x_{2}$ is chosen as a representative with probability $n_{r} / n$, and so on. We wish to know the expected time before the first $x_{i}$ is chosen as a representative. That is given precisely by the geometric distribution: the number of Bernoulli trials needed to get one success. The mean of a Bernoulli distribution with parameter $p=n_{r} / n$ is $1 / p=n / n_{r}$. Hence $\mathbb{E}|B(q, \gamma)|=n / n_{r}$, which is $O\left(\sqrt{n} / c^{3 / 2}\right)$ for the standard parameter setting.

We note that a high-probability version of the above claim follows easily from standard concentration bounds. We also point out that the expectation is over randomness in the algorithm; we are not making any distributional assumptions on the database.

After computing the nearest neighbor to the query $q$ among the representatives, the exact search algorithm uses $\gamma$ (三 $\left.\rho\left(q, r_{q}\right)\right)$ as an upper bound on the distance to $q$ 's NN to prune out some representative sets. In particular, any representative $r$ with radius $\psi_{r}$ satisfying

$$
\rho(q, r) \geq \gamma+\psi_{r}
$$

cannot possibly own $q$ 's NN. Additionally, the algorithm can safely prune out any representative $r$ such that

$$
\rho(q, r)>3 \gamma
$$

This property follows from Lemma 1. In the following we only work with inequality (4). The simultaneous use of both inequalities improved the empirical performance, but it is not necessary for the following theory.

We now estimate how many representatives violate (4) and bound how many points these representatives own. We show that all examined database points belong to a ball $B(q, 7 \gamma)$, which we subsequently bound the cardinality of.

Claim 2. The nearest neighbor of $q$ lies inside of the ball $B(q, 7 \gamma)$.

Proof. Clearly, each representative $r$ violating (4) lies inside of $B(q, 3 \gamma)$, and the NN of $q$ will appear on one of the lists $L_{r}$. If the algorithm examined every $x \in L_{r}$, it would be guaranteed to find the nearest neighbor, but we cannot say how many total points it will examine. However, the algorithm does not need to examine the entire list, as we now show.
Suppose that $x$ is $q$ 's NN; what is the maximum distance it can be from its representative $r$ ? From the triangle inequality, $\rho(x, r) \leq \rho(x, q)+\rho(q, r)$. But since $x$ is $q$ 's NN, and since $r \in X, \rho(x, q) \leq \gamma$. The other term is bounded by $3 \gamma$ on account of (4). Hence the nearest neighbor $x$ must lie within $4 \gamma$ of its representative.

Since any considered representative $r$ satisfies $\rho(q, r) \leq 3 \gamma$ (by (4)) and the NN $x$ of $q$ is within $4 \gamma$ of $r$, the triangle inequality implies that $\rho(q, x) \leq 7 \gamma$.

We have shown that the search algorithm only needs to compute distances from $q$ to points $x$ which are within distance $4 \gamma$ of their representative. Hence, if the lists $L_{r}$ are stored in sorted order according to the distance to $r$, the search algorithm can simply ignore all points $x$ more than distance $4 \gamma$ from their representative ${ }^{2}$

Finally, we bound the expected number of examined points. From Claim 2, all examined points lie in $B(q, 7 \gamma)$. Applying the expansion rate condition, we have that

$$
|B(q, 7 \gamma)| \leq|B(q, 8 \gamma)| \leq c^{3}|B(q, \gamma)| .
$$

From Claim 1 $\mathbb{E}|B(q, \gamma)|=n_{r} / n$, which we can plug into (5). As each $x$ only appears on one list $L_{r}$, each $x$ is only compared to $q$ once, implying that (5) bounds not only the cardinality of the examined set points, but also the number of computations (in the second step).

Putting everything together, we have the following theorem.

THEREOM 1. The expected number of points examined in the second stage of the exact search algorithm is $c^{3} n / n_{r}$, which is $O\left(c^{3 / 2} \sqrt{n}\right)$ for the standard parameter setting.

Since the time for the first brute force step was also $O\left(c^{3 / 2} \sqrt{n}\right)$, we have shown that the expected runtime of the exact search algorithm is $O\left(c^{3 / 2} \sqrt{n}\right)$.

\subsection{One-Shot Search}

The one-shot search algorithm is considerably simpler than the exact search algorithm, and also uses a slightly different data structure configuration. In particular, the algorithm searches only a single representative list per query, and the ownership lists of the RBC will usually overlap. Unlike the exact search algorithm, the one-shot algorithm only returns the NN with high probability, similar to locality sensitive hashing 16 .

With these differences in mind, the resulting time complexity bound is actually quite similar to the bound in Theorem 1] Recall that there are two parameters governing its run time: $n_{r}$, the number of representatives; and $s$, the number of points assigned to each representative. Hence the time complexity of the one-shot search algorithm is $O\left(n_{r}+s\right)$. The following theorem describes the setting of these parameters to guarantee a high probability of success.

Thereom 2. Set the parameters

$$
n_{r}=s=c \sqrt{n} \cdot \sqrt{\ln \frac{1}{\delta}} .
$$

Then the one-shot algorithm returns the correct $N N$ with probability at least $1-\delta$.

\footnotetext{
${ }^{2}$ For the purpose of scheduling on some systems, it may be advantageous to compute how much of each list $L_{r}$ must be explored before the second brute force operation begins. This can be done in time $O(\log n)$ per list.
} 


\begin{tabular}{lll} 
Name & Num pts & Dim \\
\hline Bio & $200 \mathrm{k}$ & 74 \\
Covertype & $500 \mathrm{k}$ & 54 \\
Physics & $100 \mathrm{k}$ & 78 \\
Robot & $2 \mathrm{M}$ & 21 \\
TinyIm & $10 \mathrm{M}$ & $4-32$
\end{tabular}

Table 1: Overview of data sets.

The proof is in Appendix B

Hence the time complexity of the one-shot algorithm is $O(c \sqrt{n})$ times a factor dependent on the desired success rate. Notice that the dependence on $c$ is lower for the one-shot algorithm than the exact algorithm; this reduced complexity seems to be reflected in the experiments.

\section{EXPERIMENTS}

We perform several sets of experiments to demonstrate the effectiveness of our methods. The first, and probably most important, set of experiments demonstrate the performance benefit of the RBC on a 48-core machine, as compared to a brute force implementation ( $\$ 7.2$. These experiments show that the RBC significantly reduces the work required for NN search and that it parallelizes effectively.

The second set of experiments demonstrates that the RBC is effective on graphics hardware (\$7.3). It is challenging to deploy data structures on such hardware, but very important because of the ubiquity of GPUs in scientific and database systems.

The final set of experiments compares the performance of the RBC to the Cover Tree on a desktop machine (\$7.4). These experiments demonstrate that the exact search algorithm is competitive with the state-of-the-art even on a machine with a low degree of parallelism.

\subsection{Experimental setup}

Our CPU implementation of the RBC was written in $\mathrm{C}$ and parallelized with OpenMP. Our GPU implementation was written in C and CUDA. Both are available for download from the author's website.

In very low-dimensional spaces, basic data structures like kd-trees are extremely effective, hence the challenging cases are data that is somewhat higher dimensional. We experiment on several different data sets over a range of dimensionalities. Table 1 provides a quick overview; we describe a few details next.

The Bio, Covertype, and Physics data sets are standard benchmark data sets used in machine learning and are available from the UCI repository 13. They have been used to benchmark NN search previously $2,7,25]$. The Robot data was generated from a Barret WAM robotic arm; see 22]. The TinyIm data set is taken from the Tiny Images database, which is used for computer vision research 28. We took the image descriptors and reduced the dimensionality using the method of random projections ${ }^{3}$ We experimented with dimensionalities of $4,8,16,32$. For all experiments, we measured distance with the $\ell_{2}$-norm (i.e. standard Euclidean distance), which is appropriate for this data.

\footnotetext{
${ }^{3}$ This dimensionality reduction technique approximately preserves the lengths of vectors, and hence is a useful preprocessor for NN search; see e.g. [21]. The technique is formally justified by the Johnson-Lindenstrauss Lemma 17.
}

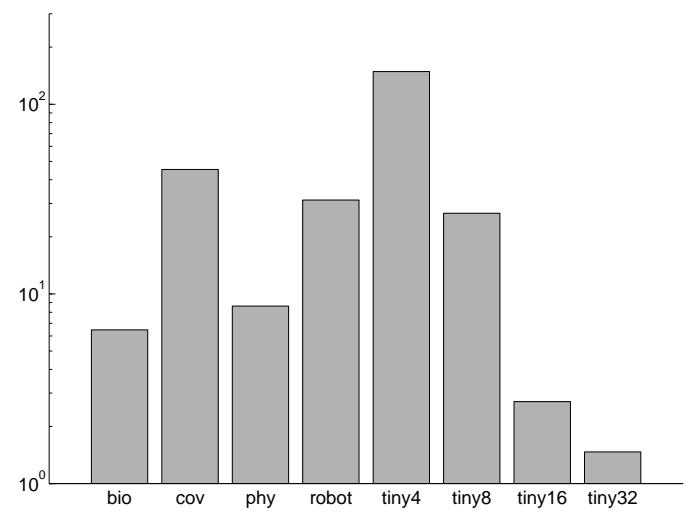

Figure 2: Speedup of exact search over brute force.

We perform the first set of CPU experiments on a 48core/4-chip AMD server machine. Each chip is a 12-core AMD 6176SE processor, and is divided into two 6-core segments. This machine has a high core count, so it is a good system to test the scalability of our algorithms.

We compare to the Cover Tree on a quad-core Intel Core i5 machine, which is a reasonable representative for a midrange desktop.

Our GPU experiments are run on a NVIDIA Tesla c2050 graphics card. This card is well-suited to general and scientific computation as it has somewhat more memory than standard graphics cards (3GB), and provides general-purpose architectural features such as error-correcting memory and caching ${ }^{4}$ We previously described the details of our GPU implementation in a workshop paper 6].

\subsection{8-core experiments}

We compare the performance of our methods to brute force search on the 48-core machine. As far as we know, there is no readily available accelerated NN method for such a machine. Furthermore, brute force is already quite fast because of the raw computational power.

First, we look at the performance of the exact search algorithm, which is guaranteed to return the exact NN. Figure2 shows the results. We are getting a strong speedup of up to two orders of magnitude, despite the challenging hardware setting and the reasonably high dimensionality of the data ${ }^{5}$

Next we consider the one-shot search algorithm. As developed in the theory section, we set $n_{r}$ (the number of representatives) and $s$ (the number of points owned per representative) equal to one another. The parameter allows one to trade-off between the quality of the solution and time required; we scan over this parameter to show the tradeoff. This algorithm is not guaranteed to return a nearest neighbor, so we must evaluate the quality of the solution. A standard error measure is the rank of the returned point: i.e., the number of database points closer to the query than the returned point 25]. A rank of 0 denotes the exact $\mathrm{NN}$, and rank of 1 denotes the second $\mathrm{NN}$, and so on.

\footnotetext{
${ }^{4}$ On the other hand, in limited experiments with (much cheaper) consumer-grade GPUs, we noticed that the runtimes were not dramatically different.

${ }^{5}$ There is one parameter in this algorithm; namely, the number of representatives chosen. The retrieval times were not particularly sensitive to this choice; see Appendix C for a detailed examination of the parameter setting.
} 

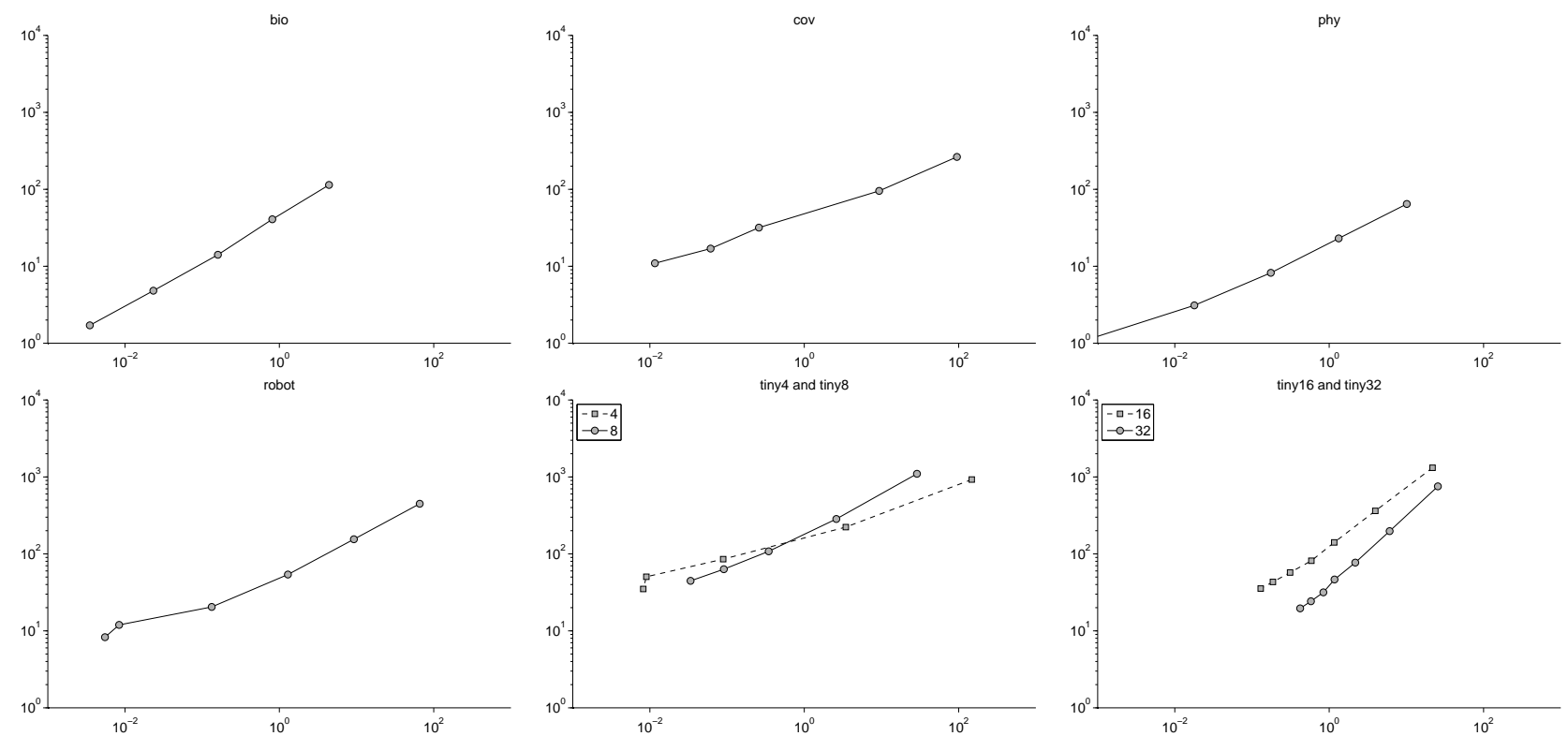

Figure 1: Results of the one-shot algorithm. This is a log-log plot of the speedup as a function of the error rate. The $x$-axis is logarithmic and runs from $10^{-3}$ to $10^{3}$ and signifies the average (over queries) rank of the returned result. For example, a rank of $10^{\circ}$ indicates that on average the algorithm returns the 2nd NN. The $y$-axis is also logarithmic and runs from $10^{0}$ (no speedup) to $10^{4}$ (10000x speedup).

Figure 1 shows the results. The speedup achieved in these experiments is quite significant; even with a rank around $10^{-1}$ (very close to exact search), the worst speedup is an order of magnitude. In applications where a small amount of error is tolerable, the one-shot search algorithm can provide a massive speedup, even better than our exact search algorithm in some cases. In many applications, e.g. in data mining, there is considerable uncertainty associated with the data, so a small amount of error in the NN retrieval is not important.

\subsection{GPU experiments}

GPUs have impressive brute force search performance 14 . However, the GPU architecture makes efficient data structure design quite difficult. In particular, GPUs are vectorstyle processors with limited branching ability; hence conditional computation typically seriously under-utilizes these devices.

We show that our RBC one-shot algorithm provides a substantial speedup over the already-fast brute force search on a GPU. Table 2 shows the results. We show only the speedups, as the error rate is the same as that of the CPU experiments. The parameter was set to achieve an error rate of roughly $10^{-1}$ (refer back to Figure 1). Our method is clearly very effective in this setting; despite the challenging hardware design, it provides a one-to-two order of magnitude speedup on all datasets.

\subsection{Cover Tree comparison}

Finally, we investigate the performance of the RBC on a quad-core desktop machine. We compare to the Cover Tree, which has state-of-the-art empirical performance on a sequential machine, and which was developed under the same notion of intrinsic dimensionality as the RBC.

This is a challenging comparison for the RBC, as the sys-

\begin{tabular}{ll} 
Data & Speedup \\
\hline Bio & 38.1 \\
Covertype & 94.6 \\
Physics & 19.0 \\
Robot & 53.2 \\
TinyIm4 & 188.4
\end{tabular}

Table 2: GPU results: speedup of the one-shot algorithm over brute force search (both on the GPU).

tem does not require the extreme constraints under which the $\mathrm{RBC}$ was designed. In particular, this system can branch effectively and has a low core count. Hence such a system can handle an algorithm with a much more complex computational structure, like the Cover Tree search algorithm. Suprisingly, the performance of the RBC is at or near stateof-the-art even in this setting.

The available implementation of the Cover Tree is singlecore [2], and a naive parallelization would not significantly benefit it. In particular, one could split the database into $p$ chunks (one for each core), run $p$ Cover Tree searches in parallel, and then perform a $p$-way reduce. However, since the dependence of the Cover Tree on the number of data points is only $O(\log n)$, doing so would only decrease the runtime from $O\left(c^{6} \log n\right)$ to, at best, $O\left(c^{6} \log \frac{n}{p}\right)$, which is a very minor improvement. Hence we run the Cover Tree only on one core, but allow the RBC to use the whole machine.

Table 3 shows the results ${ }^{6}$ The RBC is competitive on all of the datasets, and significantly outperforms the Cover Tree on the three largest datasets. Again, these results are surprising, as our exact search algorithm is much simpler

\footnotetext{
${ }^{6}$ We were unable to get the Cover Tree software to run on the full TinyIm data sets, so we reduced the database size to $1 \mathrm{M}$.
} 


\begin{tabular}{lll} 
Data & Cover Tree & RBC \\
\hline Bio & 18.9 & 6.4 \\
Covertype & 0.4 & 1.1 \\
Physics & 1.9 & 1.7 \\
Robot & 4.6 & 5.1 \\
Tiny4 & 0.5 & 1.2 \\
Tiny8 & 14.6 & 3.3 \\
Tiny16 & 178.9 & 25.1 \\
Tiny32 & 387.0 & 67.9
\end{tabular}

Table 3: Comparison of the Cover Tree and the exact RBC algorithm on a quad-core desktop machine. Times shown are the total query time in seconds for 10k queries.

than the Cover Tree search algorithm, and since our methods have the additional (significant) constraint that they must work on highly parallel systems.

We note that the RBC has a significantly lower theoretical dependence on the dimensionality than the Cover Tree $\left(O\left(c^{3 / 2}\right)\right.$ vs $\left.O\left(c^{6}\right)\right)$. This is reflected in the experiments; the two datasets that the Cover Tree significantly outperforms the RBC on are very low-dimensional: the Tiny 4 data set is four-dimensional, and the Covertype dataset has low intrinsic dimensionality 2]. This reduced dependence on dimensionality appears to be another advantage of the RBC and would be interesting to explore in future work.

\section{CONCLUSION}

In this paper, we introduced techniques for metric similarity search on parallel systems. In particular, we demonstrated that the RBC search algorithms significantly reduce the work required for NN retrieval, while being structured in such a way that can be easily implemented on parallel systems. Our experiments show that these techniques are practical on a range of modern hardware. The theory behind the RBC shows that the data structure is broadly effective.

Our code is available for download. These implementations supply additional low-level details on implementing the RBC. Moreover, they are practical tools for many NN search problems.

An interesting direction for future work is to explore the performance of the RBC in a distributed or multi-GPU environment. The RBC data structure suggests a simple distribution of the database according to the representatives that could be quite effective in such environments. There are many interesting details for study here, such as I/O and communication costs, and the connection to distributed programming paradigms. Furthermore, a distributed implementation would be broadly useful in practice.

\section{REFERENCES}

[1] S. Arya, D. M. Mount, N. S. Netanyahu, R. Silverman, and A. Wu. An optimal algorithm for approximate nearest neighbor searching. Journal of the ACM, 45(6):891-923, 1998.

[2] A. Beygelzimer, S. Kakade, and J. Langford. Cover trees for nearest neighbor. In Proceedings of the International Conference on Machine Learning, 2006.

[3] S. Brin. Near neighbor search in large metric spaces. In Proc. International Conference on Very Large Data Bases, 1995.
[4] J. Buhler. Efficient large-scale sequence comparison by locality-sensitive hashing. Bioinformatics, 17(5):419-28, 2001.

[5] B. Bustos, O. Deussen, S. Hiller, and D. Keim. A graphics hardware accelerated algorithm for nearest neighbor search. In Computational Science $\hat{a} \breve{A} S I C C S$, volume 3994 of LNCS, pages 196-199. Springer, 2006.

[6] L. Cayton. A nearest neighbor data structure for graphics hardware. In First International Workshop on Accelerating Data Management Systems Using Modern Processor and Storage Architectures, 2010.

[7] L. Cayton and S. Dasgupta. A learning framework for nearest neighbor search. In Advances in Neural Information Processing Systems 20, 2007.

[8] E. Chávez, G. Navarro, R. Baeza-Yates, and J. L. Marroquín. Searching in metric spaces. ACM Comput. Surv., 33(3):273-321, 2001.

[9] K. L. Clarkson. Nearest neighbor queries in metric spaces. Discrete and Computational Geometry, 22:63-93, 1999.

[10] K. L. Clarkson. Nearest-neighbor searching and metric space dimensions. In Nearest-Neighbor Methods for Learning and Vision: Theory and Practice. 2006.

[11] W. Dong, Z. Wang, W. Josephson, M. Charikar, and K. Li. Modeling LSH for performance tuning. In Proc. $A C M$ conference on Information and Knowledge Management (CIKM), 2008.

[12] D. L. Donoho. High-dimensional data analysis: The curses and blessings of dimensionality. Aide-memoire; AMS Conference on Math Challenges of the 21st century, 2000.

[13] A. Frank and A. Asuncion. UCI machine learning repository, 2010.

[14] V. Garcia, E. Debreuve, and M. Barlaud. Fast k nearest neighbor search using GPU. In $C V P R$ Workshop on Computer Vision on GPU (CVGPU), 2008.

[15] N. K. Govindaraju, B. Lloyd, W. Wang, M. Lin, and D. Manocha. Fast computation of database operations using graphics processors. In Proceedings of ACM SIGMOD, pages 215-226. ACM Press, 2004.

[16] P. Indyk and R. Motwani. Approximate nearest neighbors: towards removing the curse of dimensionality. In Proc. Symposium on Theory of Computing (STOC), 1998.

[17] W. B. Johnson and J. Lindenstrauss. Extensions of lipschitz mappings into a hilbert space. Contemporary Mathematics, 1984.

[18] D. R. Karger and M. Ruhl. Finding nearest neighbors in growth-restricted metrics. In Proc. Symposium on Theory of Computing (STOC), 2002.

[19] C. Kim, J. Chhugani, N. Satish, E. Sedlar, A. D. Nguyen, T. Kaldewey, V. W. Lee, S. A. Brandt, and P. Dubey. FAST: fast architecture sensitive tree search on modern CPUs and GPUs. In Proceedings of ACM SIGMOD, 2010.

[20] R. Krauthgamer and J. R. Lee. Navigating nets: simple algorithms for proximity search. In Proc. Symposium on Discrete Algorithms (SODA), 2004.

[21] T. Liu, A. W. Moore, A. Gray, and K. Yang. An investigation of practical approximate neighbor 
algorithms. In Advances in Neural Information Processing Systems, 2004.

[22] D. Nguyen-Tuong and J. Peters. Using model knowledge for learning inverse dynamics. In Proc. IEEE International Conference on Robotics and Automation, 2010.

[23] S. Omohundro. Five balltree construction algorithms. Technical report, ICSI, 1989.

[24] D. Patterson. The trouble with multicore. IEEE Spectrum, July 2010.

[25] P. Ram, D. Lee, H. Ouyang, and A. Gray. Rank-approximate nearest neighbor search: Retaining meaning and speed in high dimensions. In Advances in Neural Information Processing Systems 22, 2009.

[26] S. T. Roweis and L. K. Saul. Nonlinear dimensionality reduction by locally linear embedding. Science, 2000.

[27] J. B. Tenenbaum, V. de Silva, and J. C. Langford. A global geometric framework for nonlinear dimensionality reduction. Science, 2000.

[28] A. Torralba, R. Fergus, and W. T. Freeman. 80 million tiny images: a large dataset for non-parametric object and scene recognition. IEEE Transactions on Pattern Analysis and Machine Intelligence, 2008.

[29] E. Vidal. An algorithm for finding nearest neighbours in (approximately) constant average time. Pattern Recognition Letters, 4:145-157, 1986.

[30] R. Weber, H.-J. Schek, and S. Blott. A quantitative analysis and performance study for similarity-search methods in high-dimensional spaces. In Proc. International Conference on Very Large Data Bases, 1998.

[31] P. N. Yianilos. Data structures and algorithms for nearest neighbor search in general metric spaces. In Symposium on Discrete Algorithms, 1993.

\section{APPENDIX}

\section{A. PROOF OF LEMMA}

We restate Lemma 1 and prove it.

Lemma 2. Let $R \subset X$ and assign each $x \in X$ to its nearest $r \in R$. Let $\gamma=\min _{r \in R} \rho(q, r)$ (i.e., $\gamma$ is the distance to $q$ 's $N N$ in $R$ ). Then, if some $r^{*} \in R$ owns the nearest neighbor to $q$ in $X$, it must satisfy

$$
\rho\left(q, r^{*}\right) \leq 3 \gamma \text {. }
$$

Proof. Suppose that $x$ is $q$ 's NN in $X$-i.e. $\rho(q, x) \leq$ $\rho(q, y)$ for all $y \in X$-and that $r^{*}$ owns $x$ ( $r^{*}$ is $x$ 's NN among $R$ ). Furthermore, let $r$ be $q$ 's NN in $R$. Since $R \subset X$ and $\rho(q, r)=\gamma, \gamma$ gives an upper bound on the distance to $q$ 's NN; hence $\rho(q, x) \leq \gamma$. Using this bound along with the triangle inequality gives $\rho(x, r) \leq 2 \gamma$, but since $\rho\left(x, r^{*}\right) \leq$ $\rho(x, r)$, we have $\rho\left(x, r^{*}\right) \leq 2 \gamma$ as well. Applying the triangle inequality to the bounds on $\rho\left(x, r^{*}\right)$ and $\rho(q, x)$ yields the lemma.

\section{B. PROOF OF THE ONE-SHOT THEOREM}

We will use the following lemma, which is well-known. We include a proof for completeness.

Lemma 3. Suppose that $\rho(q, r)=\gamma$. Then

$$
B(q, \gamma) \subset B(r, 2 \gamma) \subset B(q, 4 \gamma)
$$

Proof. Let $x \in B(q, \gamma)$. Then $\rho(x, r) \leq \rho(x, q)+\rho(q, r) \leq$ $2 \gamma$, proving the first inequality. For the second, let $x \in$ $B(r, 2 \gamma)$. Then $\rho(x, q) \leq \rho(x, r)+\rho(r, q) \leq 2 \gamma+\gamma \leq 4 \gamma$.

Now we restate and prove Theorem 2

Thereom 3. Set the parameters

$$
n_{r}=s=c \sqrt{n} \cdot \sqrt{\ln \frac{1}{\delta}} .
$$

Then the one-shot algorithm returns the correct $N N$ with probability at least $1-\delta$.

Proof. If a query $q$ lies within distance $\psi_{r} / 2$ of a representative $r$, then its nearest neighbor $x_{q}$ is guaranteed to be in $L_{r}$. This follows simply from the triangle inequality:

$$
\begin{aligned}
\rho\left(r, x_{q}\right) & \leq \rho(r, q)+\rho\left(q, x_{q}\right) \\
& \leq \rho(r, q)+\rho(q, r) \quad\left(\text { since } x_{q} \text { is } q \text { 's NN }\right) \\
& \leq \psi_{r} .
\end{aligned}
$$

Thus the algorithm fails only if $\rho(q, r)>\psi_{r} / 2$ for all $r$ (and in particular that nearest one). We bound the probability of this occurring.

Let $\gamma=\rho(q, r)$, where $r$ is $q$ 's NN. Again, assume that $\gamma>\psi_{r} / 2$. We have that $B\left(r, \psi_{r}\right) \subset B(r, 2 \gamma)$, and $B(r, 2 \gamma) \subset$ $B(q, 4 \gamma)$ by Lemma 3 Hence we can apply the expansion condition to get that

$$
\left|B\left(r, \psi_{r}\right)\right| \leq|B(q, 4 \gamma)| \leq c^{2}|B(q, \gamma)|
$$

In particular, $|B(q, \gamma)| \geq 1 / c^{2}\left|B\left(r, \psi_{r}\right)\right|$; of course $B\left(r, \psi_{r}\right)$ contains the points of $L_{r}$, which we chose to be $s$. Thus there are at least $s / c^{2}$ points closer to $q$ than $r$. What is the probability that none of them were chosen as representatives?

Each point is chosen with probability $n_{r} / n$, so the probability that none are chosen is

$$
\left(1-\frac{n_{r}}{n}\right)^{s / c^{2}} \leq e^{-\frac{n_{r} s}{c^{2} n}}
$$

which follows from the inequality $(1-t / r)^{r} \leq e^{-t}$. If we plug in $n_{r}=s=\sqrt{\eta c^{2} n}$, we get a probability of failure bounded by

$$
e^{-\frac{n_{r} s}{c^{2} n}}=e^{-\eta}
$$

The theorem follows by setting $\delta=e^{-\eta}$ and rearranging.

\section{EXACT SEARCH EXPERIMENTS}

There is a single parameter to set for the exact search algorithm (the number of representatives). Here we report the results over a fairly wide range of parameters to support the experimental results. Note that the search time is relatively stable to this setting. See Figure 3 for the results. 

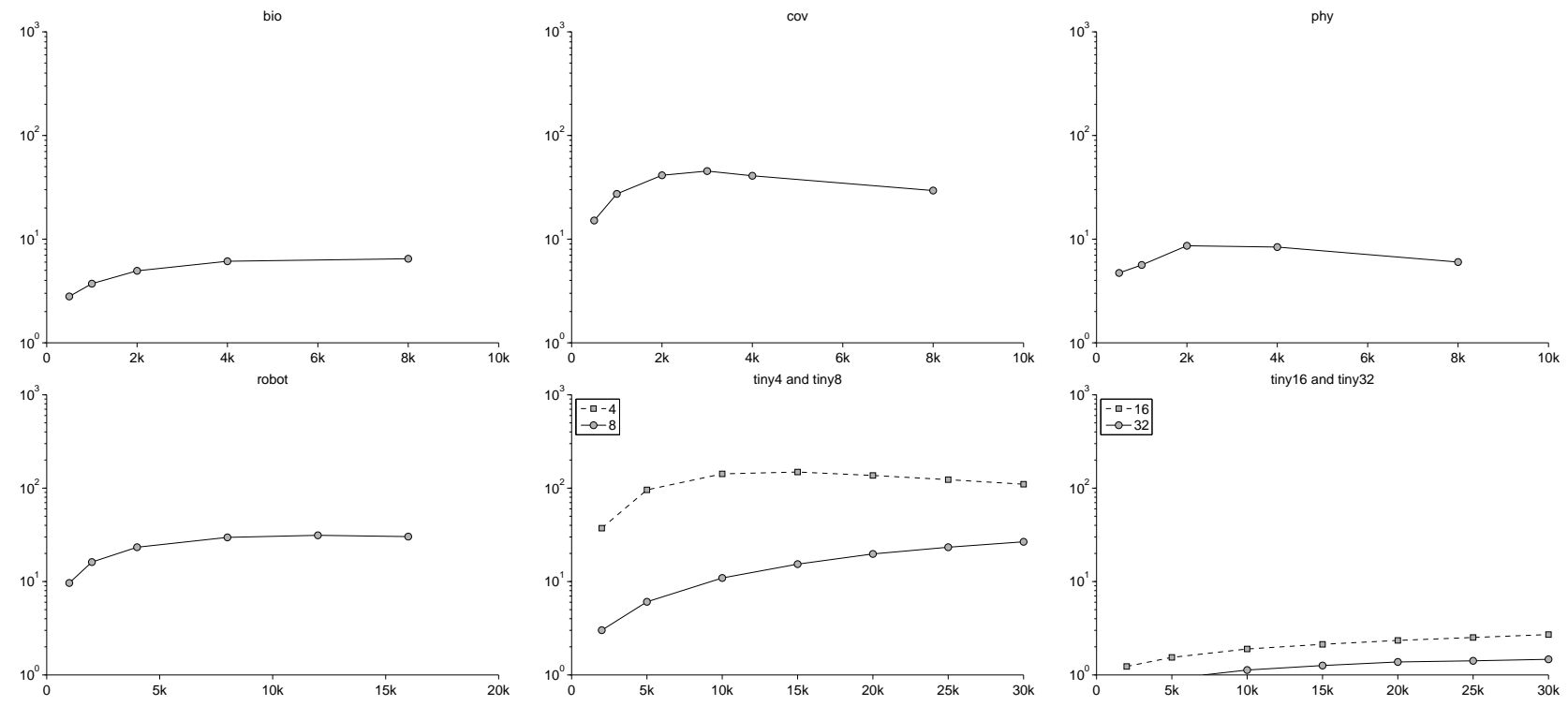

Figure 3: Results for exact search. The $y$-axis is a logarithmic scale that varies from $10^{0}$ (no speedup) to $10^{3}$ (1000x speedup). The $x$-axis is the number of representatives chosen. 